\title{
Neue Aspekte für die Sozialwirtschaft
}

VON STEFAN SCHICK UND BENJAMIN LIEDY

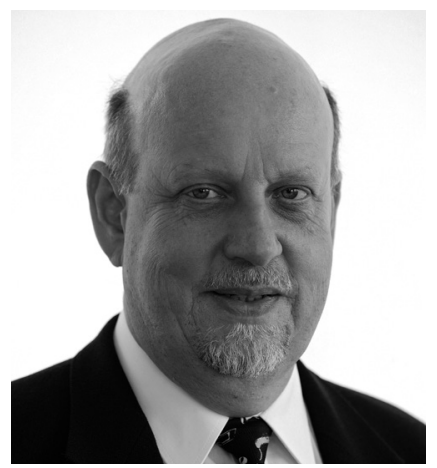

Prof. Dr. Stefan Schick ist als Rechtsanwalt und Fachanwalt für Steuerrecht in Stuttgart tätig. Vorher war er bei der Ernst \& Young AG Wirtschaftsprüfungsgesellschaft Steuerberatungsgesellschaft tätig. Er ist seit langem Mitglied des Beirats der Zeitschrift SOZIALwirtschaft und des Informationsdienstes SOZIALwirtschaft aktuell. stefan.schick@reithschick.de

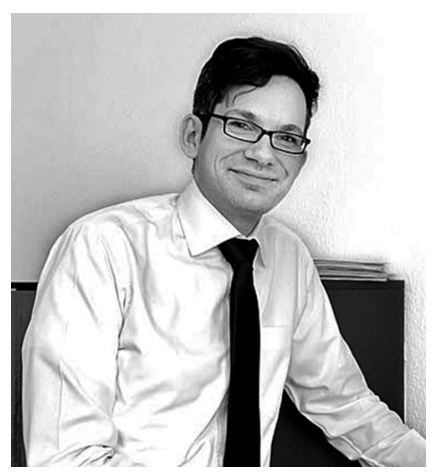

Dr. Benjamin Liedy ist Rechtsanwalt in der Kanzlei Reith Schick \& Partner, Stuttgart. Zu seinen Schwerpunkten gehört die Beratung von Non-Profit-Organisationen in vereins-, stiftungs- und gemeinnützigkeitsrechtlichen Fragen.

benjamin.liedy@reithschick.de

\author{
Aufgrund steigenden Kostendrucks werden im Non- \\ Profit-Bereich Holdingstrukturen immer häufiger. \\ Bei der Bildung solcher Unternehmensstrukturen \\ sind allerdings zahlreiche Aspekte zu beachten.
}

Der Beitrag im letzten Heft fasste die wesentlichen Motive für Holdingstrukturen in der Sozialwirtschaft zusammen und ging auf gängige Erscheinungsformen der Holding ein. Dieser Beitrag beleuchtet die gemeinnützigkeitsrechtlichen und die umsatzsteuerlichen Anforderungen, die die Gestaltung einer Holding erfüllen muss.

\section{Gemeinnützigkeit}

Um einen Mitteltransfer zwischen den einzelnen beteiligten Rechtsträger zu ermöglichen, wird in der Regel deren Gemeinnützigkeit angestrebt. Damit stellt sich zunächst die Frage nach dem gemeinnützigen Zweck der Holding.

Soll eine Managementholding realisiert werden, so dürfen operative Aktivitäten nur von sehr untergeordnetem Umfang sein. Dies - wie auch die größtmögliche Freiheit bei der Finanzierung - ist dann gewährleistet, wenn die Holding zumindest auch Mittelbeschaffungskörperschaft im Sinne der Abgabenordnung ist $(\mathbb{S} 58 \mathrm{Nr} .1 \mathrm{AO})$. Sie verfolgt dann nicht unmittelbar und operativ gemeinnützige Zwecke; ihre Tätigkeit ist beschränkt auf die Mittelbeschaffung für steuerbegünstigte Zwecke, insbesondere die Förderung der Konzerngesellschaften. Nachteilig ist allerdings, dass die reine Mittelbeschaffung keine unternehmerische Betätigung darstellt, wie sie für die umsatzsteuerliche Organschaft erforderlich ist.

Die Holding kann ihre Gemeinnützigkeit aber auch dadurch erreichen, dass sie sich eines Dritten als Hilfsperson bedient. Dies kann dadurch erfol- gen, dass sich die Holding ihrer Tochtergesellschaften, auf deren Tätigkeit sie intensiv Einfluss nimmt, als Hilfspersonen bedient. Bis zur Änderung des Anwendungserlasses zur Abgabenordnung (AEAO) konnte durch solche Gestaltungen zwar die Gemeinnützigkeit der Holding erreicht werden. Waren die Tochtergesellschaften selbst gemeinnützig, so bildete allerdings deren Tätigkeit als Hilfsperson bei ihnen keine eigene gemeinnützige Tätigkeit(vgl. Schick DB 2008, S. 893, 895 ff.). Nach der Änderung des AEAO ist die Tätigkeit der Hilfsperson, wenn sie damit auch die eigenen satzungsmäßigen Zwecke verfolgt und bei der Ausführung ein eigener Verantwortungsbereich verbleibt, selbst steuerbegünstigte Tätigkeit.

Damit kann die Gemeinnützigkeit der Holding gegebenenfalls auch dadurch erreicht werden, dass sich die Holding der Tochtergesellschaften als Hilfspersonen bedient. Allerdings wird auch dadurch nicht Unternehmenseigenschaft der Holding erreicht, die für die Begründung einer umsatzsteuerlichen Organschaft erforderlich ist.

Es verbleiben als Lösungen, um sowohl die unternehmerische Betätigung als auch die Gemeinnützigkeit zu erreichen, die Übernahme einer operativen gemeinnützigen Tätigkeit durch die Holding - dies ist im Hinblick auf eine angestrebte Ausgestaltung als Managementholding kontraproduktiv - sowie die Ausgestaltung als Mittelbeschaffungskörperschaft oder die Einschaltung der Tochtergesellschaften als Hilfspersonen und gleichzeitig die entgeltliche Erbringung von Leistungen 
der Stabs- und Zentralabteilungen der Holding an die Tochtergesellschaften.

Die entgeltliche Erbringung von Verwaltungsleistungen durch die Holding an die Tochtergesellschaften bei einem Verzicht auf eine operative gemeinnützige Tätigkeit der Holding war in der Vergangenheit deshalb gemeinnützigkeitsrechtlich problematisch, weil auf der Grundlage der Geprägetheorie die Gefahr bestand, dass die Verwaltungsleistungen der Holding das Gepräge gegeben hätten (vgl. Hauke/Tybussek NWB 2012, S. 718, 723). Nach Aufgabe der Geprägetheorie ist dagegen nur noch entscheidend, dass die Erbringung der Verwaltungsleistungen nicht den Selbstzweck der Holding bildet, sondern sich dem gemeinnützigen Zweck - der Förderung der Tochtergesellschaften funktional unterordnet.

\section{Umsatzsteuer}

Gemeinnützigkeitsrechtlich wird damit eine reine Managementholding, die keinerlei eigene operative Tätigkeit hat, relativ einfach möglich. Allerdings ist zu beachten, dass bei in der Sozialwirtschaft tätigen Trägern meist nur umsatzsteuerbefreite Umsätze generiert werden, die einen Abzug der auf die bezogenen Leistungen entfallenden Vorsteuer ausschließen. Dies bedeutet, dass eine zusätzlich entstehende Umsatzsteuer neue Kosten auslöst und damit vermieden werden muss.

Das Entstehen einer zusätzlichen Umsatzsteuerbelastung im Sozialkonzern kann dadurch ausgeschlossen werden, dass zwischen den einzelnen Konzernunternehmen und im Verhältnis zur Holding als Mutter die Voraussetzungen einer umsatzsteuerlichen Organschaft geschaffen werden. Diese setzt Folgendes voraus:

1.

Die Unternehmenseigenschaft der

- Holding: Dazu muss die Holding entgeltliche Leistungen erbringen, die Leistungserbringung beispielsweise an Tochtergesellschaften reicht dafür allerdings aus. Da sich nach Aufgabe der Geprägetheorie die Leistungserbringung im Rahmen der gemeinnützigen Betätigung der Holding - Förderung der Tochtergesellschaften - unterordnen muss und der Umfang der Leistungserbringung gemeinnützigkeitsrechtlich nicht mehr von Bedeutung ist, kann sich die unter- nehmerische Betätigung auf die Weiterberechnung von Leistungen der Stabsund Zentralabteilungen der Holding an die Tochtergesellschaften beschränken.

2. Die wirtschaftliche Eingliede- rung: Die wirtschaftliche Betätigung von Holding und Tochtergesellschaften müssen sich gegenseitig fördern und ergänzen. Nach den rechtlichen Vorgaben der Europäischen Union müssen entgeltliche Leistungen erbracht werden, die beim Leistungsempfänger zu Kostenelementen des eigenen Unternehmens werden.

2. Die finanzielle Eingliederung der 3. Tochtergesellschaften in das Unternehmen der Holding: Diese wird in der Regel sichergestellt über eine Mehrheitsbeteiligung der Holding, zumindest durch eine Stimmrechtsmehrheit der Holding in der Gesellschafterversammlung der Tochtergesellschaften.

4.

Die organisatorische Eingliede4. rung der Tochtergesellschaften in das Unternehmen der Holding: Diese wird im Regelfall dadurch erreicht, dass sich Vorstandsmitglieder oder Geschäftsführer der Holding in der Geschäftsführung der Tochtergesellschaften unmittelbar durchsetzen können. Aus steuerlicher Sicht ist die ganze oder zumindest teilweise Personenidentität der Idealfall.

\section{Gestaltungsüberlegungen}

Zunächst ist festzuhalten, dass die Holding - sollte sie gemeinnützig sein - vor der Änderung des AEAO grundsätzlich ein eigenes, operatives, gemeinnütziges Geschäft (steuerbegünstigter Zweckbetrieb) benötigte. Dies ist nunmehr nicht mehr erforderlich, so dass eine reine Managementholding, die zentrale Aufgaben für den ganzen Konzern wahrnimmt und dafür Entgelte oder Konzernumlagen berechnet, möglich geworden ist.

Für die Ausgestaltung der Führungsstruktur bestehen - sofern eine umsatzsteuerliche Organschaft angestrebt wird - die folgenden Gestaltungsansätze:

- Wird eine zentrale Steuerung angestrebt, so lässt sich dies im Rahmen einer ganzen oder teilweise personenidentischen Besetzung der
Führungsorgane von Holding und Tochtergesellschaften erreichen. Die Vorstandsmitglieder oder Geschäftsführer der Holding müssen sich in der Geschäftsführung der Tochtergesellschaften durchsetzen können. Ihre Aufgabe kann sich im Rahmen der Geschäftsführung der Tochtergesellschaften auf die Beteiligung an grundsätzlichen und strategischen Entscheidungen beschränken.

- Wird eine dezentrale Steuerung angestrebt, so lässt sich dies zunächst nur sehr bedingt mit den Anforderungen vereinbaren, die sich ergeben, wenn eine umsatzsteuerliche Organschaft angestrebt wird. Der Abschluss eines Beherrschungsvertrags anstelle der Mitgliedschaft von Vorstandsmitgliedern oder Geschäftsführern der Holding in der Geschäftsführung der Tochtergesellschaften begründet zwar keine unmittelbare Verlustausgleichspflicht der Holding, nach allgemeinen gesellschaftsrechtlichen Grundsätzen besteht aber eine Haftung der Holding als Kompensation für die gesellschaftsrechtliche Berechtigung, unmittelbar in die Geschäftsführung der Tochtergesellschaften eingreifen zu können. Denkbar ist es allerdings, dass anstelle der Mitgliedschaft von Vorstandsmitgliedern oder Geschäftsführern der Holding in der Geschäftsführung der Tochtergesellschaften die Geschäftsführer der Tochtergesellschaften eine leitende Funktion, etwa vergleichbar der eines Prokuristen, und einen Anstellungsvertrag bei der Holding erhalten.

\section{Resümee}

Die Änderung des Anwendungserlasses zur Abgabenordnung (AEAO) hat die Gestaltungsspielräume deutlich erweitert. Ob und gegebenenfalls welche Holdinggestaltung realisiert wird, ist eine Frage des Einzelfalls.

Den Vorteilen einer Holdingstruktur stehen ein Gestaltungsmehraufwand und durch die rechtlich gegliederte Struktur auch ein Verwaltungsmehraufwand gegenüber. Vor diesem Hintergrund ist in jedem Falle die Prüfung der Frage zu empfehlen, ob die mit einer Holdinggestaltung verfolgten Ziele nicht auch im Rahmen einer entsprechenden Organisationsstruktur erreicht werden können. 